1 Title:

2 Biochemical Constituents and in Vitro Antioxidant Potential of fermented wheat grains

3 using Bacillus subtilis

4 Muhammad Zahaib Ilyas ${ }^{1}$, Muhammad Roman ${ }^{2}$, Muhammad Waqas Ali ${ }^{3 *}$, Muhammad

5 Nadeem $^{4}$

$6{ }^{1}$ Department of Agronomy, Faculty of Agriculture, University of Agriculture Faisalabad, 38000,

$7 \quad$ Pakistan

$8{ }^{2}$ School of Soil and Water Conservation, Beijing Forestry University, Beijing, China

$9{ }^{3}$ School of Applied Biosciences, Kyungpook National University, Daegu 41566, Korea

$10{ }^{4}$ Soil and Water Testing Laboratory Hafizabad, Punjab, Pakistan

$11 *$ Correspondence author

12 Muhammad Waqas Ali

13 School of Applied Biosciences, Kyungpook National University, Daegu 41566, Korea

14 E-Mail: waqasali3515@yahoo.com

15 ORCID: 0000-0003-2826-4545 
16

17

18

19

20

21

22

23

24

25

26

27

28

29

30

Keywords: Antioxidant activity; phenolic contents; fermentation; wheat; minerals

Title:

\section{Biochemical Constituents and in Vitro Antioxidant Potential of fermented wheat grains using Bacillus subtilis}

High antioxidants level in food is gradually becoming popular because of the enhanced risk of oxidative stress in humans. Bread wheat is rich in vital antioxidants but its major bioactive compounds are not available for the human. This study was conducted with the aim to enhance the phytochemical constituents and antioxidative activity of wheat grains by fermenting it with the use of Bacillus subtilis KCTC 13241. The antioxidative potential was determined by DPPH (2,2diphenyl-1-picryl- hydrazyl) and ABTS (3-ethyl-benzothiazo- line-6-sulfonic acid) radical scavenging assay as well by the concentration of amino acids, flavonoids, minerals, carbohydrates and phenolic compounds. Different varieties showed different free radical scavenging potential on fermentation, which was significantly high with respect to their corresponding unfermented wheat varieties. The highest potential was found in a fermented wheat variety named as Namhae and this combination can be used in pharmaceutical and food industries. 


\section{Introduction}

Globally, cereals are the most important part of the human diet and source of protein, carbohydrate, fibers, minerals and vitamins. Processing of cereal's products is important both for nutritive and sensorial properties. Microbial fermentation, conversion of complex organic molecules into simpler ones like amino acids, peptides etc., has been commonly used to improve the nutritional quality of foodstuff (Liu et al. 2017). These peptides and amino acids are potent natural antioxidants (Dordević et al. 2010). Consumption of food products manufactured by using a whole grain of cereals, unfermented, has been coupled with some chronic diseases like diabetes and cancer etc. (Wojdyło et al. 2007).

Wheat is a staple food of the people of temperate regions and ranks as second in overall production after maize. It contains numerous phenolics namely vanillic, caffeic, ferulic, salicylic, p-coumaric, gentisic, sinapic acids and syringic (Naczk and Shahidi 2006). Polyphenolics have diverse biological properties including anti-oxidant, anti-inflammatory and anti-microbial properties (Trouillas et al. 2003). In cereals, mostly, phenolics acids are not present in free form but as conjugates with proteins, sugars and fatty acids. So, the hydrolysis process must be adopted to get more phenolics in the cereal grains (Wojdyło et al. 2007). Some studies reported that total phenolics content significantly enhanced by microbial fermentation (Cai et al. 2012). However, other hydrolysis processes as an enzymatic reaction are not economically feasible because the synthetic enzymes involved in this process are not economically viable.

Fermentation with Bacillus subtilis has been employed for making proteinases in past few decades worldwide (Juan and Chou 2010). Anti-oxidants derived from a plant with metal chelating and free-radical scavenging properties can minimize the risk of oxidative stress and keep a balance 
54 between anti-oxidants and oxidants in the human body (Bhanja et al. 2009). Over the past few

55 decades, phenolics have potentially been used to prevent different chronic disease including

56 cancer, diabetes mellitus, cardiovascular disease, neurodegenerative diseases and osteoporosis

57 (Martins et al. 2011). In microbial fermentation, microorganisms release a number of

58 carbohydrases like xylanase, cellulases, esterase and pectinases convert unavailable phenolics to

59 available form (Bhanja et al. 2009).

Naturally, wheat grains have a sufficient quantity of anti-oxidants but human body unable

61 to use these anti-oxidants because of the presence of these compounds in bound form. So instead

62 of getting health benefits by eating these food items, people suffer from health complications. The

63 main objective of this research is to find a potent bacterial strain by employing wheat grain in order

64 to enhance the antioxidant properties and nutrient status of wheat grain to avoid these health

65 complications. Bacillus subtilis has been employed for fermentation but a few results about its 66 activity are available.

\section{Materials and methods}

68

69

70

71

72

73

74

\section{Reagents}

Acetonitrile, HPLC grade water and methanol were bought from Fisher Scientific (Fairlawn, OH, USA). Ferric chloride, 2,4,6-tripyridyl-s-triazine, sodium acetate, rutin, glacial acetic acid, 2,2azinobis (3-ethyl-benzothiazoline-6-sulfonic acid), Folin-Ciocalteu phenol reagent and potassium per sulfate were purchased from Sigma Chemical Co. (St. Louis, MO, USA). All chemicals were of analytical grade. 


\section{Microorganism and inoculum preparation}

76 Formerly isolated and purified strain, B. subtilis KCTC 13241 was employed in the fermentation

77 of wheat grains. Under laboratory conditions, B. subtilis was retained on the slants of nutrient agar.

78 For the preparation of inoculums, the activated culture of microbes was streaked on the slants of

79 nutrient agar (as a medium) and incubated, precisely, at $27^{\circ} \mathrm{C}$ for 24 hours. The newly grown

80 active cells were collected in sterilized distilled water. After adjusting the level $7.43 \log \mathrm{CFU} \mathrm{mL}^{-}$

$81{ }^{1}$ and this suspension were utilized for the microbial fermentation of cooked wheat grains (Ali et 82 al. 2018).

\section{Preparation of fermented wheat}

84 There were four varieties of wheat used in this experiment named as Baekjoong, Jeokjoong, 85 Milseoung and Namhae. Twenty grams of wheat grain were taken in Erlenmeyer flasks of $250 \mathrm{~mL}$, mixed with twenty $\mathrm{mL}$ of distilled water, steamed at $130{ }^{\circ} \mathrm{C}$ for twenty minutes and eventually

87 cooled to room temperature. Steamed substrates were separately incubated with bacterial 88 suspension, mixed thoroughly and incubated at $25^{\circ} \mathrm{C}$ for 3 days named as fermented wheat (FW), 89 secondly, the same inoculated wheat varieties were frozen instead of incubation and named as 90 cooked wheat $(\mathrm{CW})$ as a control. The experiment was repeated three times.

\section{$91 \quad$ Viable cell number}

92 One gram sample plus nine millilitres of $0.85 \%$ sodium chloride solution was mixed and diluted 93 bacterial suspension was spread over agar medium, incubated for 36 hours at $27^{\circ} \mathrm{C}$ for counting 94 of colonies (Ali et al. 2017). 
96

97 Samples extraction was performed by using the protocol of $\mathrm{Xu}$ and Chang (Xu and Chang 2007)

\section{Sample extraction conditions}

with few adjustments. Samples were freeze-dried and ground by using the electric grinder. The prepared samples were extracted by taking ten grams of each sample plus one hundred millilitres of $80 \%$ of methanol (v/v) and incubated for twenty-four hours at $25{ }^{\circ} \mathrm{C}$. After incubation, the extracts were goes under centrifugation for twenty minutes at $3000 \mathrm{rpm}$. The collected supernatants were further filtered by using a PVDF filter of $0.45-\mu \mathrm{m}$ Millipore (Schleicher \& Schuell, GmbH, Dassel, Germany). The filtrates were kept at $4{ }^{\circ} \mathrm{C}$ in dark for proposed analysis.

\section{Determination of total phenolic content}

Total phenolics were estimated by the following protocol of Folin-Ciocalteu (Katsube et al. 2004).

The methanolic extracts were filtered by a $0.2 \mu \mathrm{m}$ syringe filter (Water, Milford, MA, USA). 50 mL methanolic extract plus one millilitre of two percent $\mathrm{Na}_{2} \mathrm{CO}_{3}$ (sodium carbonate) was mixed and left for three minutes. Then mixed with fifty $\mu \mathrm{L}$ of one normal Folin-Ciocalteu phenol reagent and left for thirty minutes at $25^{\circ} \mathrm{C}$ in the dark. The samples were run on a spectrophotometer (Thermal Fischer Scientific, Vantaa, Finland) to read absorbance on $750 \mathrm{~nm}$ wavelength against a blank sample. The concentration of total phenolics was computed by employing standard calibration curve, which was plotted by utilizing gallic acid, and stated as $\mu \mathrm{gGAE} / \mathrm{g}$ grain (microgram gallic acid equivalent per gram of wheat grains).

\section{Evaluation of total flavonoid content}

It was determined by the method reported by Adhikari (Adhikari et al. 2018). Fermented wheat grains extract was added into an equal proportion of deionized water and then thirty-five 
117 microliters of $5 \%$ sodium nitrate $\left(\mathrm{NaNO}_{3}\right)$ solution was added and allowed to react for five minutes

118 at $25{ }^{\circ} \mathrm{C}$ followed by the addition of seventy microliters of $10 \%$ aluminium chloride $\left(\mathrm{AlCl}_{3}\right)$.

119 Addition of $175 \mathrm{~mL}$ of one molar sodium hydroxide $(\mathrm{NaOH})$ was done after five minutes and

120 absorbance was recorded instantly $505 \mathrm{~nm}$ by using spectrophotometer (Thermo Fischer Scientific,

121 Vantaa, Finland). A calibration curve was drawn by using catechin as a standard and total

122 flavonoids stated as catechin equivalent $(\mu \mathrm{gCE} / \mathrm{g})$.

123 DPPH (2,2-diphenyl-1-picrylhydrazyl) radical-scavenging assay

124 The free radical-scavenging activity of fermented wheat extracts was measured by using protocol 125 illustrated by Bilal (Bilal et al. 2016) with slight modifications. 0.1 mM DPPH solution was freshly 126 prepared in $99.9 \%$ pure methanol which was then used for the analysis. An equal amount of 127 methanolic extracts plus $0.1 \mathrm{mM}$ freshly prepared DPPH solution was mixed properly and kept for 128 half an hour in the incubator to measure absorbance at $516 \mathrm{~nm}$ and Trolox was treated as a control.

129 The following equation was used to calculate the DPPH radical-scavenging activity and expressed 130 in percentage.

131 DPPH radical-scavenging activity $(\%)=[1-(A b S-A b C)] \times 100$

132 Where $A b S$ was the absorbance of the test compound, $A b C$ was the absorbance of control.

133 ABTS cation radical-scavenging assay

134 It was measured by following the protocol illustrated by Bilal (Bilal et al. 2016). Potassium 135 persulphate was used for the oxidation of ABTS to generate $\mathrm{ABTS}^{\bullet+}$ in the solution. It was diluted 136 before use to attain an absorbance of $0.7 \pm 0.02$ at $735 \mathrm{~nm}$ by using $50 \%$ ethanol. The ABTS 137 reagent was added in the sample, mixed vigorously and absorbance was recorded at $735 \mathrm{~nm}$ after 
138 three minutes of mixing to measure the scavenging activity. Fifty percent ethanol was used as a

139 blank and Trolox as a positive control. The following equation was used to calculate the ABTS

140 radical-scavenging activity.

141 ABTS cation radical-scavenging activity $(\%)=[1-(A b S-A b C)] \times 100$

\section{Free amino acids composition}

143 It was determined by following the protocol by Ali (Ali et al. 2017). One gram of ground, the

144 sieved sample was hydrolyzed with six molar $\mathrm{HCl}$ for 24 hours at $105^{\circ} \mathrm{C}$ to determine amino acid

145 profile through Hitachi Amino Acid Analyzer (L-8900, Hitachi, Japan). Standard amino acid

146 mixture solution (Type H, Wako Pure Chemical Industries Ltd., Japan) was used for the

147 determination of endogenous amino acid profile. Samples from all treatment were analyzed in

148 triplicate and stated as mg per gram of dry weight.

149 Analysis of minerals and carbohydrates

150 Minerals were identified and quantified by (Andualem and Gessesse 2014) method with slight 151 modifications. Fifteen milliliters of HNO3 was added into $0.5 \mathrm{~g}$ of the freeze-dried sample of FW 152 and $\mathrm{CW}$. An equal volume of distilled water was put for dilution. Plasma-atomic emission 153 spectrometer (ICP AES: Varian Vista, Varian Australia, Victoria, Australia) was used to determine 154 the concentrations of different minerals. Standards for each mineral were used for calibration of 155 the instrument.

156 The carbohydrates of the samples was identified and quantified according to the method followed 157 by Kang (Kang et al. 2014). Ground dried samples were homogenized with liquid nitrogen and 158 sugar extracted with aqueous ethanol. The ethanol was evaporated by rotary evaporator. The 
159 residues were dissolved in water and filter was injected to HPLC Waters system (Millipore Crop,

160 Waters Chromatography, Milford, MA, USA) and the sugar signals were detected by Waters

161 refractive index detector. The de-ionized water was used as mobile phase and the flow rate was

$1620.5 \mathrm{ml} / \mathrm{min}$ at $90{ }^{\circ} \mathrm{C}$. Glucose and fructose were measured on the basis of peak areas and 163 comparison with a calibration curve obtained with the corresponding standards.

164 Statistical Analysis

165 Recorded data were subjected to ANOVA (analysis of variance) by employing SAS version 9.3

166 (SAS Institute Inc., Cary, NC, USA). The treatment means separation was done by DMRT

167 (Duncan's multiple range tests) at the 95\% confidence level. The results were stated as the means

$168 \pm \mathrm{SD}$ (standard deviation) of three replicates.

\section{Results and discussion}

\section{Variation in viable microbial population in fermented and cooked wheat}

171 The ability of four different fermented wheat (FW) to support viable microbial cells is shown in

172 fig. 1. Among four varieties of wheat, the descending order of bacterial population (log CFU/g)

173 was recorded in FW as Namhae (13.05 \pm 1.13), Milseoung (12.76 \pm 1.65), Baekjoong (12.34 \pm 174 1.34) and Jeokjoong $(11.27 \pm 0.92)$ but in cooked wheat $(\mathrm{CW})$, the highest population was recorded

175 in the Namhae $(8.53 \pm 1.04)$ and lowest was in the Jeokjoong $(6.32 \pm 0.43)$. The microbial 176 population recorded in Baekjoong and Milseoung was $7.45 \pm 0.76$ and $7.98 \pm 0.61$ respectively.

177 The variation in microbial population within the same factor perhaps due to the difference in 178 nutritional status and size of wheat grains under study and between the factors due to change in 179 the time span of incubation. Results of the previous experiment showed that viable bacterial 
180 population increased significantly after fermentation (Cho et al. 2011) which is also proved by the 181 present study.

\section{Total phenolic content (TPC) of fermented and cooked wheat}

The total phenolic contents of the fermented wheat varieties and their corresponding cooked wheat varieties are demonstrated in fig. 2. Phenolic compounds are crucial in the food-related fields because of their ability to reduce the process of lipid peroxidation (Wojdyło et al. 2007). The TPC $\left(\mu \mathrm{g} \mathrm{GAEg}^{-1}\right)$ that was measured in four fermented as well as cooked wheat varieties showed that the highest TPC was available in fermented wheat (FW) varieties and their concentrations compared with CW from highest to lowest was as Namhae FW (1671.32 \pm 32.54$)$, CW (1139.57 $\pm 23.01)$, Milseoung FW (1597.43 \pm 10.87), CW (1106.96 \pm 9.43), Baekjoong FW (1533.78 \pm 20.39), CW (1054.21 \pm 12.17$)$ and Jeokjoong FW (1321.46 \pm 19.17) CW (954.43 \pm 14.54$)$. The results of TPC showed that amount of free phenolic compounds were considerably increased according to the potential of above wheat varieties when these were allowed to ferment for 72 hours then cooked wheat. Previous experimental results also narrated that the phenolic content of different cereals was significantly increased when fermented and highest was noted in buckwheat (Dordević et al. 2010). In cereals, fermentation led to break down of cell walls causing synthesis as well as the liberation of various bioactive compounds (Katina et al. 2007) and the above statement is also supported by the experiment. The above results also showed the fermentation potential of B. subtilis KCTC 13241 for these four wheat varieties. Previous results revealed that the concentration of available TPC increases in fermented cereals and the extent of increase depends on the species of microorganism (Kariluoto et al. 2006). 


\section{Total flavonoid contents (TFC) of fermented and cooked wheat}

203 Flavonoids could be used to suppress different diseases including neurodegenerative disorders, 204 cancer as well as cardiovascular diseases (Cai et al. 2012). Consumption of vegetables and fruits 205 rich in flavonoids has been associated with defence against heart diseases and cancer (Juan and 206 Chou 2010). The difference in TFC of FW and corresponding CW is shown in fig. 3. All the wheat 207 varieties that were fermented had shown a greater concentration of total flavonoids then its 208 corresponding cooked variety. Namhae (FW) had maximum flavonoids content with $142.65 \pm 2.25$ and its corresponding Namhae (CW) showed $76.33 \pm 6.31$ followed by Milseoung (FW) with

$210137.26 \pm 6.73$ TFC and its corresponding Milseoung $(\mathrm{CW})$ had $65.96 \pm 4.76 \mathrm{TFC}$ then Baekjoong 211 (FW), which had 124.57 \pm 7.94 TFC compared to Baekjoong (CW) with TFC of 51.29 \pm 4.41 . The 212 lowest concentration of TFC was found in the Jeokjoong (FW) with $109.21 \pm 5.11$ and its 213 corresponding had TFC of $43.87 \pm 3.27$ which is significantly less than FW and it is also supported 214 by the outcomes of past study which narrated that fermented soybeans extract was highly 215 concentrated in TFC than its corresponding cooked soybeans extract (Juan and Chou 2010). The 216 difference in TFC of FW and CW might be owing to the difference in genetic potential of wheat 217 varieties and it is referred by Bilal et al. (2016). Another study showed that the concentration of 218 TFC in soybean significantly enhanced when fermented using microorganisms (Lee et al. 2008). 219 Low TFC in cooked wheat is due to lack of fermentation with bacteria, which causes degradation 220 of complex, bound and unavailable phytochemicals that scavenge free radicals and protect humans 221 from oxidative stress. $\mathrm{Xu}$ and Change (2007) also reported the similar results that fermented or 222 flavonoids rich foods reduce the chance of human diseases related to oxidative stress (Xu and 223 Chang 2007). 


\section{DPPH free radical scavenging activity}

226 The DPPH free radical scavenging assay has been commonly used to measure the radical 227 scavenging potential owing to ease and high accuracy of this method (Dordević et al. 2010). The 228 free radicals of DPPH have been employed in the estimation of free radical scavenging potential 229 of different plants extract (Katalinic et al. 2006). The DPPH free radical scavenging potential of 230 the four FW and CW varieties is shown in fig. 4. The scavenging potential of FW and $\mathrm{CW}$ extract 231 was expressed in percentage and highest percentage was observed in Namhae (FW) with $44.36 \pm$ $232 \quad 2.15(\%)$ as compared to cooked Namhae (control) with very low scavenging activity of $27.43 \pm$ $2330.84(\%)$ followed by the Milseoung (FW) with $39.03 \pm 1.56(\%)$ of scavenging potential which 234 was a valuable difference from its corresponding cooked Milseoung (control) whose scavenging 235 potential was $25.88 \pm 1.23(\%)$. The DPPH free radical scavenging potential of Baekjoong (FW) 236 extract was $35.71 \pm 1.39(\%)$ but its corresponding control (cooked Baekjoong) showed a very 237 weak potential to scavenge radical of DPPH that was $23.04 \pm 1.45(\%)$. Among these treatments, 238 the lowest potential was noticed in case of Jeokjoong (FW) extract with $32.01 \pm 0.99(\%)$ but in 239 comparison with its corresponding control $(20.61 \pm 1.76)$, it showed a considerable potential to 240 scavenge DPPH radicals. Other investigations also demonstrated that some wheat varieties have 241 weak scavenging potential (31\%) for DPPH radicals ( $\mathrm{Yu}$ et al. 2002). There is a strong 242 antioxidative potential of fermented Namhae according to the results of our experiment and also 243 reported by the Dordecic et al., (2010) who worked on cereals (Dordević et al. 2010). It is cleared 244 that fermented Namhae has enough potential to protect humans against the oxidative stress but in 
245 comparison with the antioxidative potential of legumes, wheat is placed in lower rank and this is

246 also mentioned by Acosta-Estrada et al., (2014) (Acosta-Estrada et al. 2014).

\section{ABTS radical scavenging potential of FW and CW extract}

248 The $\mathrm{ABTS}^{\bullet+}$ scavenging potential of FW and CW is shown in the fig. 5. The ABTS free radical 249 scavenging assay has been widely used for the determination of the antioxidative potential of food 250 products (Bilal et al. 2016). Free radicals scavenging compounds have more potential to scavenge 251 ABTS $^{-+}$than DPPH free radical (Sachindra and Bhaskar 2008). The similar case was also found 252 in the following results of our research. The highest $\mathrm{ABTS}^{\circ+}$ scavenging potential (expressed in $253 \%)$ showed by the Namhae (FW) was $53.94 \pm 2.39$ followed by Milseoung (FW) with 47.19 \pm 2.87 254 then Baekjoong (FW) with $42.45 \pm 2.06$ and Jeokjoong (FW) with least antioxidative potential of $25538.83 \pm 1.45$; the antioxidative potential of their corresponding controls $(\mathrm{CW})$ was $36.49 \pm 2.23$, $25633.28 \pm 1.65,31.17 \pm 1.27$ and $29.86 \pm 1.04$ respectively. There was a significant increase in the 257 antioxidant potential of fermented seed extract as compared to unfermented seed extract which 258 was also pronounced by the Starzynska-Janiszewska et al., (2008) (Starzyńska-Janiszewska et al. 259 2008). The above experimental results were also proof of the results explained by the Dey and 260 Kuhad (2014) in the case of wheat (Dey and Kuhad 2014).

\section{Amino acid profile of FW and CW}

262 The concentration of amino acids available in the FW and CW varieties is presented in table 1. 263 An enzyme released by the Bacillus Subtilis hydrolyzes the protein sources present in the wheat 264 into a free amino acid and the short peptide that then instantly available to the human body (Liu et 265 al. 2017). A quantitative study of fourteen amino acids was carried out both in FW and CW in this 
266

267

268

269

270

271

272

273

274

275

276

277

278

279

280

281

282

283

284

285

286

287

experiment including seven essential amino acid namely phenylalanine, valine, threonine, methionine, leucine, isoleucine, histidine and seven non-essential amino acids namely arginine, serine, glutamic acid, glycine, alanine, aspartic acid, tyrosine. The concentration of amino acids was expressed in $\mathrm{mg} / \mathrm{g}$. The total amount of essential plus nonessential amino acids in FW decreases as Namhae $<$ Milseoung $<$ Baekjoong $<$ Jeokjoong and their corresponding concentrations are $116.71 \pm 4.64,115.91 \pm 5.17,102.78 \pm 5.32$ and $101.93 \pm 4.13$ respectively; there is a significant total amino acid difference from their corresponding CW as $95.94 \pm 5.98$, $95.33 \pm 6.76,84.02 \pm 4.87$ and $81.48 \pm 5.32$ respectively. Glutamic acid is the most abundant amino acid in FW as well as CW but the highest amount in FW (Namhae). Few amino acids like tyrosine, histidine, methionine have been described as antioxidants and especially, histidine shows strong free radical scavenging potential (Udenigwe and Aluko 2011). Amino acids have surplus electrons which can be used to scavenge free radicals. Some amino acids regulate the expression of genes, enhance skeletal muscles' growth, burn non-essential body fat (Wu 2009). Leucine, valine, isoleucine and phenylalanine are act as neurotransmitters which affect the performance of the brain and nervous system (Fernstrom 2013). A high proportion of available amino acid act as a supportive character in quality assurance of food products (Bilal et al. 2016).

\section{Minerals and carbohydrate contents in FW and CW}

Mineral contents are separated into two main categories micro and macro minerals. Calcium, potassium, sodium, phosphorous, magnesium, and sulfur are macro minerals whereas iron, selenium, copper, iodine, cobalt, zinc, chromium, molybdenum and boron are micro minerals (Lukaski, 2004). In this research, the most important ten minerals were analysed and showed in table 2. Wheat cultivars showed the interesting results related to minerals. Jeokjoong showed the 
288 highest contents of iron, calcium, magnesium and potassium in $\mathrm{CW}$ as well as after fermentation 289 in FW. Baekjoong has the other mineral contents in higher concentration such as phosphorus, 290 manganese and sodium. Overall the total minerals contents after fermentation has been increased.

291 In current study, first time minerals composition was determined in fermented wheat using Bacillus 292 Subtilis. This difference in the mineral contents might be due to the interaction of bacterial 293 fermentation with other metabolites breakdown (Maria John et al. 2015). These essential minerals 294 play an important role in the health. For nerve functioning, blood clotting and immune system, 295 calcium plays its role for their functions and also supports to relax and contract the muscles. 296 Sodium helps for regulation of electrolyte balance, heart function and metabolic activities. 297 Magnesium aids to make the proteins, immune system and control constipation while potassium 298 needs for nerve transmission, control blood pressure and muscles shriveling. Iron present in red 299 blood cells and carries oxygen in the body and helpful in metabolism (Gharibzahedi and Jafari 300 2017). The value of glucose and fructose represented in table. 2. Beakjoong has the highest value 301 of glucose $(0.46 \mathrm{~g} / 100 \mathrm{~g})$ in $\mathrm{CW}$ and this value increased after fermentation as compared to other 302 varieties. The value of fructose is higher in Jeokjoong (CW) and this value increased in Milseoung $303(90.54 \mathrm{~g} / 100 \mathrm{~g})$ after the fermentation of wheat grains. These variations in the carbohydrate 304 contents are because of potential of cultivars and due to presence of Bacillus Subtilis.

\section{Conclusion}

306 The results of the present study reveal that microbial fermentation of bread wheat using Bacillus 307 subtilis KCTC 13241 is a highly effective technique for quality enhancement both in antioxidant 308 potential and nutritional aspects. Most of the free radicals scavenging compounds are present in 309 bound form in wheat and functionally inert because they are not available to the human body. It 
310 is cleared from the obtained results that there is a significant value addition to fermented wheat

311 varieties because of increase in their potential to reduce the risk of oxidative stress as well as to

312 combat with hidden hunger due to malnutrition. In the present era, the main focus is on the increase

313 of wheat yield which leads towards the dilution in quality characters of wheat. All the time, less

314 attention is paid to utilize the quality parameters up to the potential by employing suitable

315 techniques. Considerable quality gap is found between available and potential status in

316 unfermented wheat varieties during the study. To fulfil this gap, bacterial (B. subtilis KCTC 13241)

317 fermentation is the best option that is convenient, reliable and very cheap. Therefore, it is

318 concluded that Namhae variety is the most potent regarding the discussed parameters and can be

319 used on a commercial scale for fermentation.

320 Conflict of interest

321 No conflict of interest in this research work.

322 References

323 Acosta-Estrada BA, Gutiérrez-Uribe JA, Serna-Saldívar SO (2014) Bound phenolics in foods, a

324 review. Food Chem 152:46-55. https://doi.org/10.1016/j.foodchem.2013.11.093

325 Adhikari B, Kumar Dhungana S, Waqas Ali M, et al (2018) Antioxidant activities, polyphenol, 326 flavonoid, and amino acid contents in peanut shell. J Saudi Soc Agric Sci. 327 https://doi.org/10.1016/j.jssas.2018.02.004

328 Ali M, Kim I-D, Bilal S, et al (2017) Effects of Bacterial Fermentation on the Biochemical 329 Constituents and Antioxidant Potential of Fermented and Unfermented Soybeans Using 330 Probiotic Bacillus subtilis (KCTC 13241). Molecules 22:2200. 
331

332

333

334

335

336

337

338

339

340

341

342

343

344

345

346

347

348

349

350

https://doi.org/10.3390/molecules22122200

Ali MW, Shahzad R, Bilal S, et al (2018) Comparison of antioxidants potential, metabolites, and nutritional profiles of Korean fermented soybean (Cheonggukjang) with Bacillus subtilis KCTC 13241. J Food Sci Technol 55:. https://doi.org/10.1007/s13197-018-3202-2

Andualem B, Gessesse A (2014) Proximate composition, mineral content and antinutritional factors of Brebra (Millettia ferruginea) seed flour as well as physicochemical characterization of its seed oil. Springerplus 3:298. https://doi.org/10.1186/2193-1801-3-298

Bhanja T, Kumari A, Banerjee R (2009) Enrichment of phenolics and free radical scavenging property of wheat koji prepared with two filamentous fungi. Bioresour Technol 100:28612866. https://doi.org/10.1016/j.biortech.2008.12.055

Bilal S, Khan AL, Waqas M, et al (2016) Biochemical constituents and in vitro antioxidant and anticholinesterase potential of seeds from Native Korean Persimmon Genotypes. Molesafcscules 21:. https://doi.org/10.3390/molecules21070893

Cai S, Wang O, Wu W, et al (2012) 21.Comparative Study of the Effects of Solid-State Fermentation with.pdf

Cho KM, Lee JH, Yun HD, et al (2011) Changes of phytochemical constituents (isoflavones, flavanols, and phenolic acids) during cheonggukjang soybeans fermentation using potential probiotics Bacillus subtilis CS90. J Food Compos Anal 24:402-410. https://doi.org/10.1016/j.jfca.2010.12.015

Dey TB, Kuhad RC (2014) Enhanced production and extraction of phenolic compounds from 
wheat by solid-state fermentation with Rhizopus oryzae RCK2012. Biotechnol Reports 4:120-127. https://doi.org/10.1016/j.btre.2014.09.006

Dordević TM, Šiler-Marinković SS, Dimitrijević-Branković SI (2010) Effect of fermentation on antioxidant properties of some cereals and pseudo cereals. Food Chem 119:957-963. https://doi.org/10.1016/j.foodchem.2009.07.049

Fernstrom JD (2013) Large neutral amino acids: Dietary effects on brain neurochemistry and function. Amino Acids 45:419-430. https://doi.org/10.1007/s00726-012-1330-y

Gharibzahedi SMT, Jafari SM (2017) The importance of minerals in human nutrition: Bioavailability, food fortification, processing effects and nanoencapsulation. Trends Food Sci Technol 62:119-132. https://doi.org/10.1016/j.tifs.2017.02.017

Juan MY, Chou CC (2010) Enhancement of antioxidant activity, total phenolic and flavonoid content of black soybeans by solid state fermentation with Bacillus subtilis BCRC 14715 . Food Microbiol 27:586-591. https://doi.org/10.1016/j.fm.2009.11.002

Kang SM, Radhakrishnan R, You YH, et al (2014) Phosphate Solubilizing Bacillus megaterium mj1212 Regulates Endogenous Plant Carbohydrates and Amino Acids Contents to Promote Mustard Plant Growth. Indian J Microbiol 54:427-433. https://doi.org/10.1007/s12088-0140476-6

Kariluoto S, Aittamaa M, Korhola M, et al (2006) Effects of yeasts and bacteria on the levels of folates in rye sourdoughs. Int $\mathrm{J}$ Food Microbiol 106:137-143. https://doi.org/10.1016/j.ijfoodmicro.2005.06.013 
371 Katalinic V, Milos M, Kulisic T, Jukic M (2006) Screening of 70 medicinal plant extracts for antioxidant capacity and total phenols. Food Chem 94:550-557. https://doi.org/10.1016/j.foodchem.2004.12.004

Katina K, Liukkonen KH, Kaukovirta-Norja A, et al (2007) Fermentation-induced changes in the nutritional value of native or germinated rye. J Cereal Sci 46:348-355. https://doi.org/10.1016/j.jcs.2007.07.006

Katsube T, Tabata H, Ohta Y, et al (2004) Screening for Antioxidant Activity in Edible Plant Products: Comparison of Low-Density Lipoprotein Oxidation Assay, DPPH Radical Scavenging Assay, and Folin-Ciocalteu Assay. J Agric Food Chem 52:2391-2396. https://doi.org/10.1021/jf035372g

Lee IH, Hung YH, Chou CC (2008) Solid-state fermentation with fungi to enhance the antioxidative activity, total phenolic and anthocyanin contents of black bean. Int J Food Microbiol 121:150-156. https://doi.org/10.1016/j.ijfoodmicro.2007.09.008

Liu F, Chen Z, Shao J, et al (2017) Effect of fermentation on the peptide content, phenolics and antioxidant activity of defatted wheat germ. Food Biosci 20:141-148. https://doi.org/10.1016/j.fbio.2017.10.002

Maria John KM, Enkhtaivan G, Lee J, et al (2015) Spectroscopic determination of metabolic and mineral changes of soya-chunk mediated by Aspergillus sojae. Food Chem 170:1-9. https://doi.org/10.1016/j.foodchem.2014.08.029

Martins S, Mussatto SI, Martínez-Avila G, et al (2011) Bioactive phenolic compounds: Production and extraction by solid-state fermentation. A review. Biotechnol Adv 29:365-373. 

https://doi.org/10.1016/j.biotechadv.2011.01.008

393 394 395 396 397 398 399 400 401 402 403 404 405 406 407 408 409 410 411

Naczk M, Shahidi F (2006) Phenolics in cereals, fruits and vegetables: Occurrence, extraction and analysis. J Pharm Biomed Anal 41:1523-1542. https://doi.org/10.1016/j.jpba.2006.04.002

Sachindra NM, Bhaskar N (2008) In vitro antioxidant activity of liquor from fermented shrimp biowaste. Bioresour Technol 99:9013-9016. https://doi.org/10.1016/j.biortech.2008.04.036

Starzyńska-Janiszewska A, Stodolak B, Jamróz M (2008) Antioxidant properties of extracts from fermented and cooked seeds of Polish cultivars of Lathyrus sativus. Food Chem 109:285292. https://doi.org/10.1016/j.foodchem.2007.12.028

Trouillas P, Calliste C, Allais D, et al (2003) Antioxidant,-anti-inflammatory-andantiproliferative-properties-of-sixteen-water-plant-extracts-used-in-the-Limousincountryside-as-herbal-teas.pdf. 80:399-407. https://doi.org/10.1016/S0308-8146(02)002820

Udenigwe CC, Aluko RE (2011) Chemometric analysis of the amino acid requirements of antioxidant food protein Hydrolysates. Int $\mathrm{J}$ Mol Sci 12:3148-3161. https://doi.org/10.3390/ijms12053148

Wojdyło A, Oszmiański J, Czemerys R (2007) Antioxidant activity and phenolic compounds in 32 selected herbs. Food Chem 105:940-949. https://doi.org/10.1016/j.foodchem.2007.04.038

Wu G (2009) Amino acids: Metabolism, functions, and nutrition. Amino Acids 37:1-17. https://doi.org/10.1007/s00726-009-0269-0

Xu BJ, Chang SKC (2007) A comparative study on phenolic profiles and antioxidant activities of 
412 legumes as affected by extraction solvents. J Food Sci 72:. https://doi.org/10.1111/j.1750$413 \quad 3841.2006 .00260 . x$

414 Yu L, Perret J, Davy B, et al (2002) Antioxidant properties of cereal products. J Food Sci 67:2600415 2603. https://doi.org/10.1111/j.1365-2621.2002.tb08784.x

416 
417 Table 1. The composition of free amino acids during cooked and fermented wheat.

\begin{tabular}{|c|c|c|c|c|c|}
\hline Amino Acids mg/g & Sample & Baekjoong & Jeokjoong & Milseoung & Namhae \\
\hline \multirow{2}{*}{ arginine } & CW & $4.63 \pm 0.98$ & $4.93 \pm 1.07$ & $5.35 \pm 1.34$ & $5.43 \pm 1.49$ \\
\hline & FW & $5.14 \pm 1.43$ & $5.69 \pm 1.64$ & $6.43 \pm 1.13$ & $4.76 \pm 0.45$ \\
\hline \multirow{2}{*}{ threonine } & $\mathrm{CW}$ & $2.42 \pm 0.42$ & $2.31 \pm 0.36$ & $2.65 \pm 0.33$ & $1.82 \pm 0.37$ \\
\hline & FW & $3.23 \pm 0.67$ & $3.73 \pm 0.51$ & $3.52 \pm 1.02$ & $2.39 \pm 0.62$ \\
\hline \multirow{2}{*}{ serine } & $\mathrm{CW}$ & $3.35 \pm 0.30$ & $4.13 \pm 0.86$ & $3.93 \pm 0.24$ & $4.05 \pm 0.56$ \\
\hline & FW & $4.24 \pm 0.85$ & $5.03 \pm 1.12$ & $4.37 \pm 0.53$ & $5.35 \pm 1.68$ \\
\hline \multirow{2}{*}{ glutamic acid } & $\mathrm{CW}$ & $37.98 \pm 3.86$ & $35.29 \pm 2.43$ & $38.74 \pm 4.63$ & $38.99 \pm 5.43$ \\
\hline & FW & $45.65 \pm 5.39$ & $42.32 \pm 3.78$ & $48.80 \pm 5.24$ & $47.94 \pm 4.94$ \\
\hline \multirow{2}{*}{ phenylalanine } & $\mathrm{CW}$ & $5.15 \pm 1.87$ & $5.37 \pm 1.58$ & $5.99 \pm 0.71$ & $4.65 \pm 0.92$ \\
\hline & FW & $6.76 \pm 1.74$ & $6.97 \pm 1.49$ & $6.54 \pm 1.19$ & $5.16 \pm 1.57$ \\
\hline \multirow{2}{*}{ glycine } & $\mathrm{CW}$ & $3.04 \pm 0.68$ & $3.45 \pm 0.43$ & $4.56 \pm 0.92$ & $4.81 \pm 1.03$ \\
\hline & FW & $4.72 \pm 0.65$ & $4.78 \pm 1.04$ & $5.41 \pm 1.23$ & $5.49 \pm 1.31$ \\
\hline \multirow{2}{*}{ alanine } & $\mathrm{CW}$ & $2.81 \pm 0.71$ & $2.23 \pm 0.50$ & $3.89 \pm 0.69$ & $4.82 \pm 1.04$ \\
\hline & FW & $3.46 \pm 1.13$ & $3.93 \pm 1.23$ & $4.25 \pm 1.02$ & $5.63 \pm 1.78$ \\
\hline \multirow{2}{*}{ histidine } & $\mathrm{CW}$ & $2.96 \pm 0.57$ & $3.52 \pm 1.07$ & $4.53 \pm 0.67$ & $4.21 \pm 1.18$ \\
\hline & FW & $3.13 \pm 0.59$ & $4.13 \pm 1.16$ & $5.37 \pm 0 . .48$ & $5.04 \pm 1.24$ \\
\hline \multirow{2}{*}{ valine } & $\mathrm{CW}$ & $3.59 \pm 0.12$ & $4.58 \pm 1.21$ & $5.01 \pm 144$ & $4.17 \pm 0.72$ \\
\hline & FW & $3.99 \pm 0.64$ & $5.16 \pm 1.67$ & $6.05 \pm 0.89$ & $5.99 \pm 1.13$ \\
\hline \multirow{2}{*}{ methionine } & $\mathrm{CW}$ & $1.48 \pm 0.54$ & $1.87 \pm 0.67$ & $1.95 \pm 0.59$ & $2.54 \pm 0.81$ \\
\hline & FW & $2.46 \pm 0.52$ & $2.47 \pm 0.83$ & $2.89 \pm 0.83$ & $3.69 \pm 1.48$ \\
\hline \multirow{2}{*}{ aspartic acid } & $\mathrm{CW}$ & $4.67 \pm 0.21$ & $3.62 \pm 0.16$ & $4.98 \pm 0.24$ & $5.16 \pm 0.29$ \\
\hline & FW & $5.25 \pm 1.54$ & $3.98 \pm 1.23$ & $5.23 \pm 1.38$ & $5.82 \pm 1.67$ \\
\hline \multirow{2}{*}{ isoleucine } & $\mathrm{CW}$ & $2.98 \pm 1.23$ & $3.26 \pm 0.76$ & $3.72 \pm 0.89$ & $4.14 \pm 0.51$ \\
\hline & FW & $3.87 \pm 1.71$ & $4.78 \pm 0.62$ & $4.57 \pm 0.82$ & $5.68 \pm 1.37$ \\
\hline
\end{tabular}




\begin{tabular}{|c|c|c|c|c|c|}
\hline \multirow{2}{*}{ leucine } & CW & $6.45 \pm 1.13$ & $4.33 \pm 0.31$ & $6.79 \pm 1.37$ & $7.15 \pm 0.92$ \\
\hline & FW & $7.39 \pm 1.05$ & $5.31 \pm 1.87$ & $7.75 \pm 0.95$ & $8.31 \pm 1.82$ \\
\hline \multirow{2}{*}{ tyrosine } & CW & $2.51 \pm 0.76$ & $2.86 \pm 0.63$ & $3.24 \pm 0.43$ & $3.98 \pm 0.74$ \\
\hline & $\mathrm{FW}$ & $3.49 \pm 1.15$ & $3.65 \pm 0.84$ & $4.75 \pm 1.08$ & $5.46 \pm 1.39$ \\
\hline \multirow{2}{*}{ Total Amino Acids } & CW & $84.02 \pm 4.87$ & $81.48 \pm 5.32$ & $95.33 \pm 6.76$ & $95.94 \pm 5.98$ \\
\hline & $\mathrm{FW}$ & $102.78 \pm 5.32$ & $101.93 \pm 4.13$ & $115.91 \pm 5.17$ & $116.71 \pm 4.64$ \\
\hline
\end{tabular}

418 All values are the average of determinations in three independent experiments. The data were analyzed with a one-

419 way ANOVA followed by Duncan multiple range tests $(\mathrm{P}<0.05)$. CW: cooked wheat, FW: fermented wheat

420

421

422 
423 Table 2. Minerals and carbohydrate contents during cooked and fermented wheat.

\begin{tabular}{|c|c|c|c|c|c|}
\hline Minerals $\mathrm{mg} / 100 \mathrm{~g}$ & Sample & Baekjoong & Jeokjoong & Milseoung & Namhae \\
\hline \multirow{2}{*}{ Iron } & $\mathrm{CW}$ & $3.17 \pm 0.23$ & $3.26 \pm 0.13$ & $3.25 \pm 0.14$ & $3.11 \pm 0.22$ \\
\hline & FW & $3.98 \pm 0.89$ & $4.71 \pm 0.32$ & $4.73 \pm 0.28$ & $3.26 \pm 0.85$ \\
\hline \multirow{2}{*}{ Calcium } & $\mathrm{CW}$ & $25.58 \pm 3.83$ & $28.43 \pm 3.64$ & $23.65 \pm 4.49$ & $23.89 \pm 4.63$ \\
\hline & $\mathrm{FW}$ & $29.87 \pm 2.23$ & $30.87 \pm 2.77$ & $27.35 \pm 3.43$ & $26.39 \pm 3.98$ \\
\hline \multirow{2}{*}{ Phosphorus } & $\mathrm{CW}$ & $287.77 \pm 7.32$ & $285.43 \pm 4.92$ & $278.49 \pm 4.82$ & $275.15 \pm 5.94$ \\
\hline & FW & $315.32 \pm 5.75$ & $302.54 \pm 5.98$ & $295.45 \pm 5.98$ & $300.24 \pm 6.47$ \\
\hline \multirow{2}{*}{ Magnesium } & $\mathrm{CW}$ & $120.87 \pm 6.41$ & $124.29 \pm 7.73$ & $123.99 \pm 5.13$ & $123.04 \pm 7.15$ \\
\hline & FW & $139.11 \pm 5.34$ & $145.87 \pm 9.65$ & $140.62 \pm 7.60$ & $131.82 \pm 8.73$ \\
\hline \multirow{2}{*}{ Selenium } & $\mathrm{CW}$ & $71.04 \pm 6.21$ & $70.65 \pm 3.76$ & $71.41 \pm 3.29$ & $70.25 \pm 6.37$ \\
\hline & FW & $70.65 \pm 4.19$ & $69.78 \pm 4.65$ & $72.76 \pm 7.13$ & $68.92 \pm 5.92$ \\
\hline \multirow{2}{*}{ Potassium } & $\mathrm{CW}$ & $356.22 \pm 6.94$ & $360.32 \pm 9.54$ & $351.73 \pm 7.82$ & $349.14 \pm 7.81$ \\
\hline & FW & $368.71 \pm 7.15$ & $385.12 \pm 10.07$ & $388.60 \pm 10.54$ & $365.84 \pm 8.95$ \\
\hline \multirow{2}{*}{ Manganese } & $\mathrm{CW}$ & $3.99 \pm 0.43$ & $3.28 \pm 1.09$ & $2.91 \pm 0.84$ & $2.27 \pm 0.32$ \\
\hline & FW & $4.13 \pm 0.74$ & $4.96 \pm 0.61$ & $3.65 \pm 0.73$ & $3.99 \pm 0.36$ \\
\hline \multirow{2}{*}{ Zinc } & $\mathrm{CW}$ & $1.48 \pm 0.54$ & $2.19 \pm 0.67$ & $1.95 \pm 0.59$ & $2.54 \pm 0.81$ \\
\hline & FW & $2.46 \pm 0.52$ & $2.47 \pm 0.83$ & $2.89 \pm 0.83$ & $3.69 \pm 1.48$ \\
\hline \multirow{2}{*}{ Sodium } & $\mathrm{CW}$ & $2.32 \pm 0.19$ & $2.26 \pm 0.87$ & $2.25 \pm 0.15$ & $2.30 \pm 0.95$ \\
\hline & FW & $4.78 \pm 0.84$ & $4.00 \pm 0.90$ & $4.49 \pm 0.36$ & $4.02 \pm 0.72$ \\
\hline \multirow{2}{*}{ Copper } & $\mathrm{CW}$ & $0.45 \pm 0.17$ & $0.33 \pm 0.09$ & $0.32 \pm 0.01$ & $0.39 \pm 0.43$ \\
\hline & FW & $0.73 \pm 0.07$ & $0.63 \pm 0.05$ & $0.41 \pm 0.11$ & $0.72 \pm 0.17$ \\
\hline \multirow{2}{*}{ Glucose g/100g } & $\mathrm{CW}$ & $0.41 \pm 0.06$ & $0.46 \pm 0.13$ & $0.23 \pm 0.09$ & $0.19 \pm 0.10$ \\
\hline & FW & $0.42 \pm 0.12$ & $0.65 \pm 0.17$ & $0.25 \pm 0.08$ & $0.21 \pm 0.04$ \\
\hline \multirow{2}{*}{ Fructose $\mathrm{g} / 100 \mathrm{~g}$} & $\mathrm{CW}$ & $71.02 \pm 3.27$ & $65.38 \pm 5.32$ & $67.27 \pm 4.63$ & $64.12 \pm 4.76$ \\
\hline & FW & $72.24 \pm 3.81$ & $75.42 \pm 6.87$ & $90.54 \pm 6.17$ & $76.13 \pm 5.53$ \\
\hline
\end{tabular}


424 All values are the average of three replicates. The data were analyzed with a one-way ANOVA followed by Duncan 425 multiple range tests $(\mathrm{P}<0.05)$. CW: cooked wheat, $\mathrm{FW}$ : fermented wheat

426

427

428

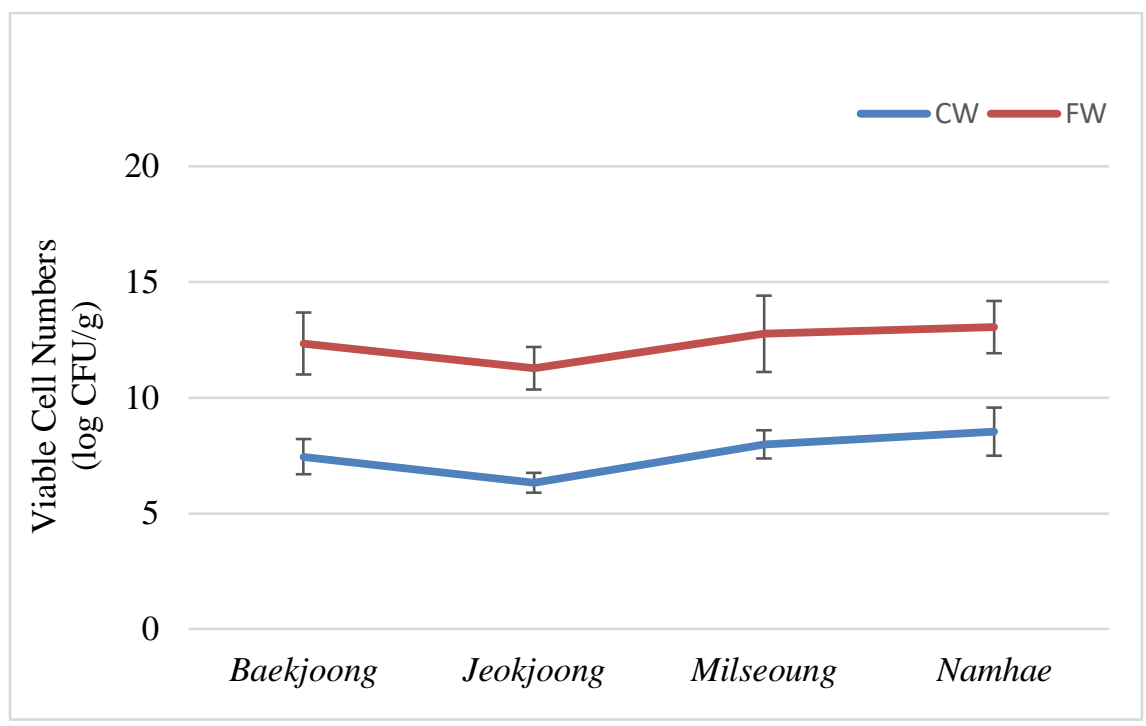

429 Fig. 1. Viable cell numbers of cooked wheat and fermented wheat. Error bars (mean \pm SD) represent significant 430 differences among the treatments. The data were analyzed with a one-way ANOVA followed by Duncan multiple 431 range tests $(\mathrm{P}<0.05)$. $\mathrm{CW}$ : cooked wheat, $\mathrm{FW}$ : fermented wheat 


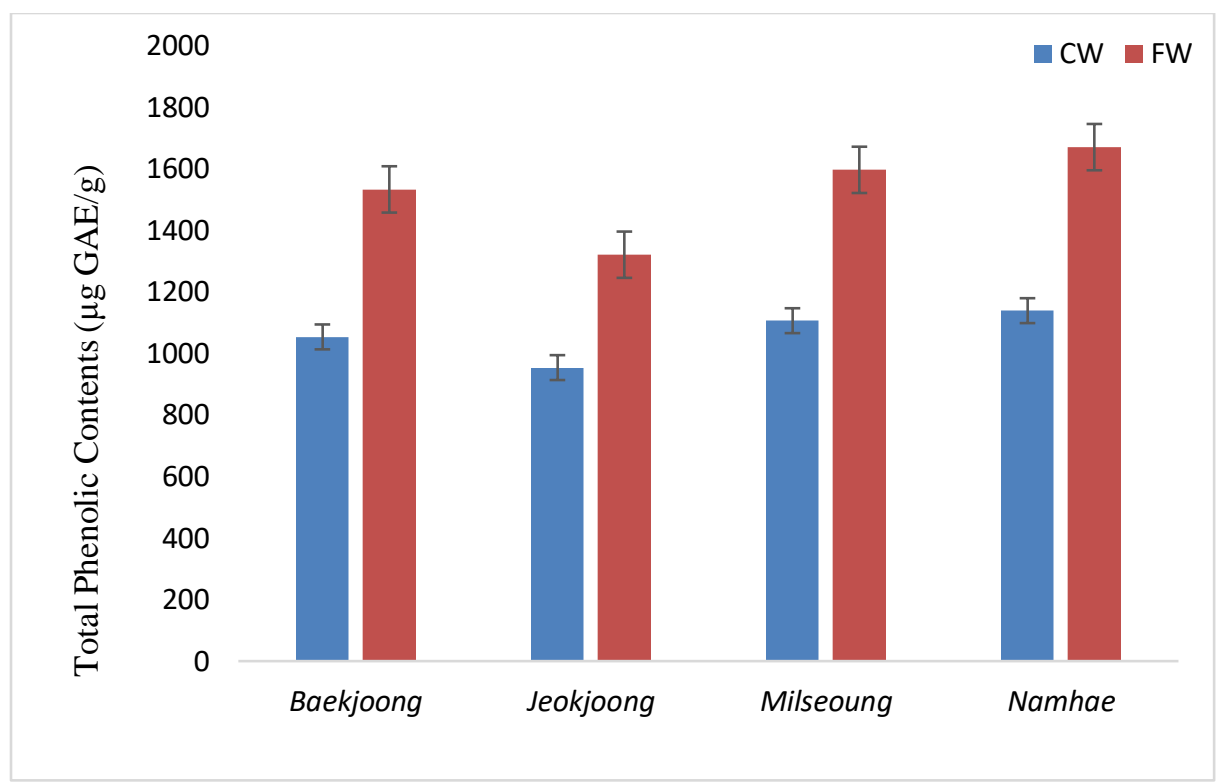

Fig. 2. Total phenolic contents of cooked wheat and fermented wheat. Error bars (mean \pm SD) represent significant differences among the treatments. The data were analyzed with a one-way ANOVA followed by Duncan multiple range tests $(\mathrm{P}<0.05)$. CW: cooked wheat, $\mathrm{FW}$ : fermented wheat

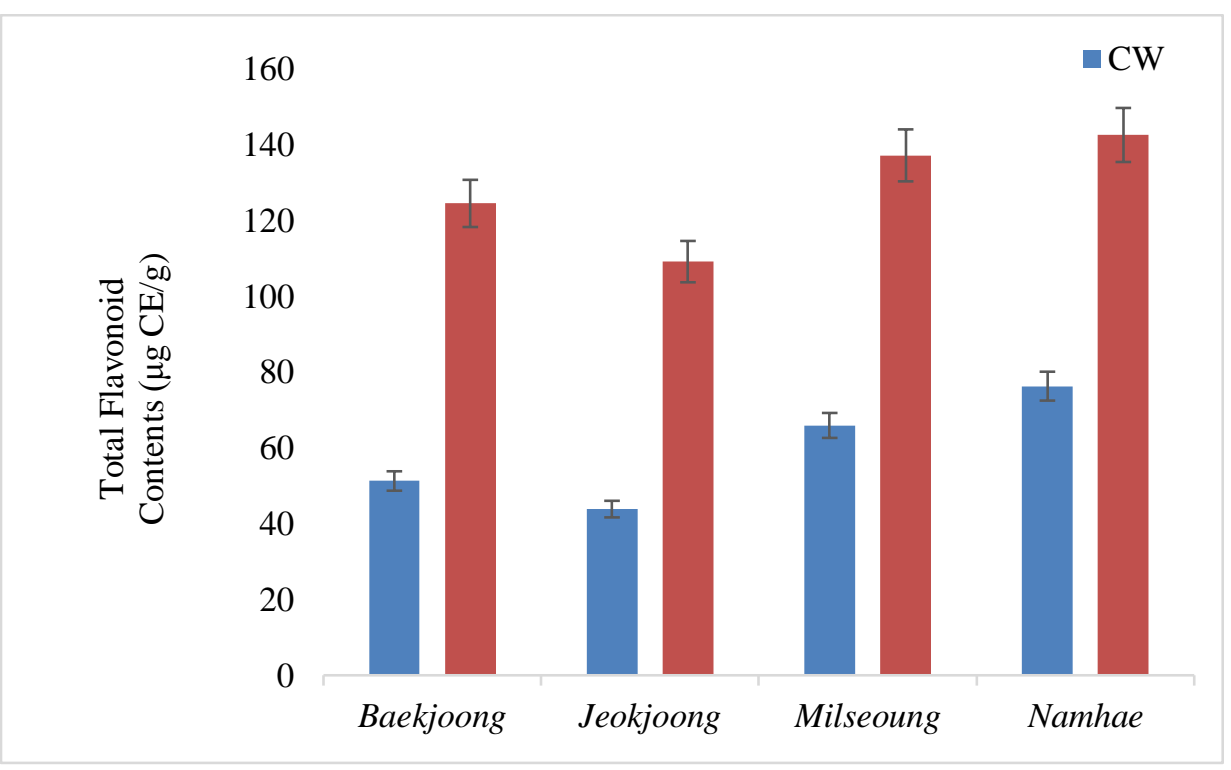

440

Fig. 3. Total flavonoid contents of cooked wheat and fermented wheat. Error bars (mean \pm SD) represent significant differences among the treatments. The data were analyzed with a one-way ANOVA followed by Duncan multiple 


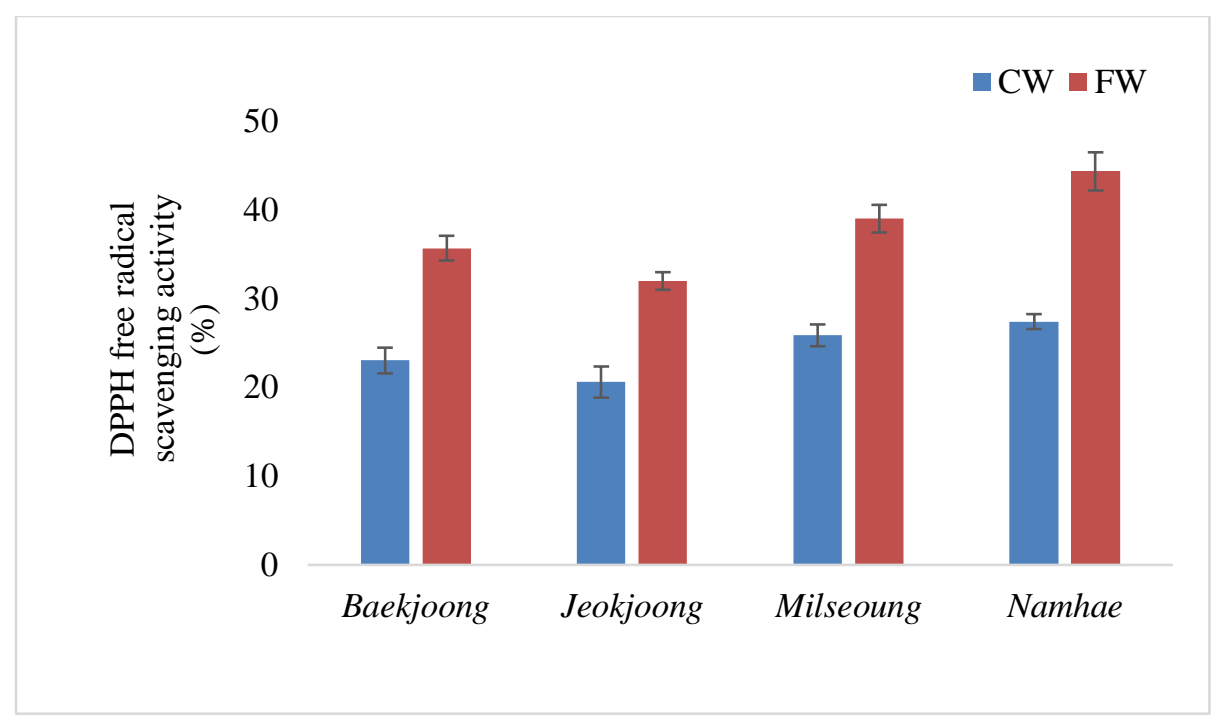

444 Fig. 4. DPPH free radical scavenging activity of cooked wheat and fermented wheat. Error bars (mean \pm SD) represent 445 significant differences among the treatments. The data were analyzed with a one-way ANOVA followed by Duncan 446 multiple range tests $(\mathrm{P}<0.05)$. CW: cooked wheat, FW: fermented wheat

447

448

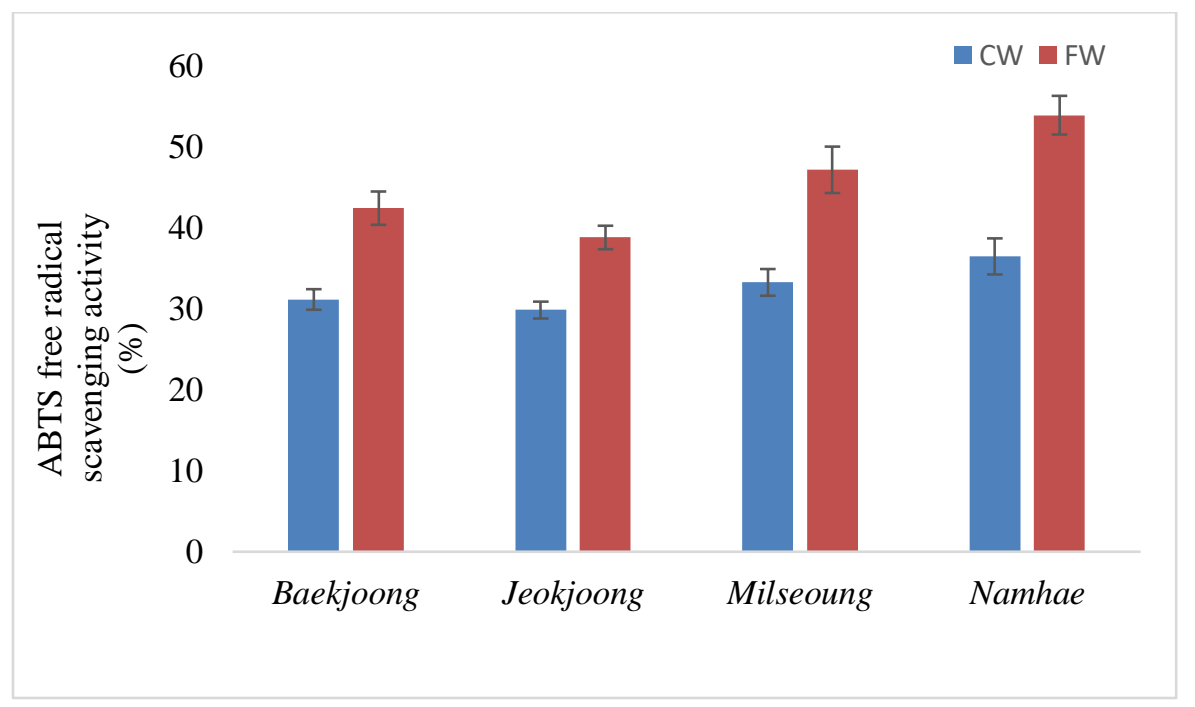

450 Fig. 5. ABTS free radical scavenging activity of cooked wheat and fermented wheat. Error bars (mean \pm SD) represent 451 significant differences among the treatments. The data were analyzed with a one-way ANOVA followed by Duncan 452 multiple range tests $(\mathrm{P}<0.05)$. CW: cooked wheat, $\mathrm{FW}$ : fermented wheat 